\title{
त्री \\ La peculiaridad del conocimiento tecnológico
}

\author{
Alberto Gupani
}

\begin{abstract}
电
RESUMEN

La filosofía de la tecnología, disciplina relativamente reciente, viene destacando la especificidad del conocimiento tecnológico, principalmente a diferencia del científico. En este artículo reúno diversas características del conocimiento tecnológico que aparecen dispersas en la bibliografía correspondiente. Mi propósito es contribuir así a una mejor comprensión de la índole de ese conocimiento como un todo y a la solución de algunas cuestiones sobre la naturaleza de la ciencia, principalmente la de su posible compromiso intrínseco con la tecnología.
\end{abstract}

Palabras-clave • Conocimiento tecnológico. Tecnología como conocimiento. Conocimiento científico. Control de la naturaleza.

La reflexión filosófica sobre la tecnología dentro de la academia es relativamente reciente. Mario Bunge, uno de sus pioneros, escribía en 1980 acerca de esos "dos vecinos que se desconocían", la filosofía y la tecnología, llamando la atención para la amplitud y la riqueza de los asuntos que un filósofo podría detectar en el mundo tecnológico (Bunge, 1980, Cap. 13). Desde aquella época, afortunadamente, el panorama cambió mucho y la tecnología es hoy objeto de examen por filósofos de variadas orientaciones.

Carl Mitcham, en un libro que constituye probablemente la mejor introducción a esta área filosófica, señala que la tecnología puede ser abordada desde cuatro perspectivas básicas: como cierto tipo de objetos (los artefactos), como una clase específica de conocimiento (el saber tecnológico), como un conjunto de actividades (resumidas en producir y usar artefactos) y como manifestación de determinada voluntad del ser humano en relación al mundo (tecnología como volición) (cf. Mitcham, 1994, p. 6o). Es claro que las cuatro perspectivas no pueden ser separadas, pero es posible prestar atención a una de ellas, preferentemente. Es lo que me propongo en este artículo, en que presento los rasgos que, en la opinión de los estudiosos, caracterizan el 
conocimiento tecnológico (sobre todo, a diferencia del científico). Para esa finalidad, escojo, entre las diversas definiciones de tecnología que se ofrecen en la bibliografía disponible, la de Mario Bunge: "el estudio científico de lo artificial". Bunge explicita: "Si se prefiere, la tecnología puede ser vista como el campo de conocimiento relativo al proyecto de artefactos y la planificación de su realización, operación, ajuste, manutención y monitoración, a la luz de conocimiento científico" (Bunge, 1985a, p. 231). Ella me parece más apropiada para analizar la dimensión cognitiva de la tecnología que otras como "una forma de conocimiento humano" dirigida a producir "objetos más y más diversificados, con rasgos cada vez más interesantes, de un modo cada vez más eficiente" (Skolimowski, 1983 [1966], p. 43-4), que no incluye alusión a la ciencia; "conocimiento de lo que funciona" (Jarvie, 1983 [1967], p. 55), que es demasiado estrecha; "implementaciones prácticas de la inteligencia" (Ferré, 1995 [1988], p. 26), demasiado amplia, o "ciencias de lo artificial" (Simon, 1981 [1969]) que parece dar por presupuesta su heterogeneidad.

El propósito de este trabajo es doble. Por un lado, y principalmente, producir una visión de conjunto de los principales argumentos que encontré, hasta el momento, sobre el asunto, pues los rasgos del saber tecnológico que aquí reúno se hallan dispersos en la literatura correspondiente. Esa visión de conjunto (aún no, propiamente, una síntesis) debe ayudar a comprender la índole del conocimiento tecnológico como un todo. Por otro lado, deseo contribuir mediante esa visión de conjunto (que incluye, como mostraré, divergencias y oscilaciones conceptuales) a la búsqueda de una respuesta para ciertas cuestiones filosóficas sobre la ciencia, tales como: ¿es la ciencia inherentemente tecnológica?, ¿qué valores comparten la ciencia y la tecnología?, ¿prueba el éxito tecnológico la verdad científica? y ¿tiene el mismo significado la palabra conocimiento en ambos campos?

"La tecnología, aunque pueda aplicar ciencia, no es lo mismo que, o enteramente ciencia aplicada."

Walter Vincenti

Para comprender el conocimiento tecnológico, la primera medida parece ser dejar de considerarlo como (mera) aplicación del conocimiento científico. En ese sentido, todos los estudiosos parecen estar de acuerdo. La identificación de la tecnología con la ciencia aplicada debe mucho a obras históricas influyentes, advierte Edwin Layton Jr., 
otro precursor de la filosofía de la tecnología (cf. Layton, 1974). ${ }^{\mathbf{T}}$ Trátase, observa Layton, de una noción que encierra toda una teoría sobre la relación ciencia-tecnología, correspondiendo a la visión de historiadores que identificaron la tecnología con técnicas y objetos producidos mediante ellas, ignorando los procesos de pensamiento implicados en tal producción. Pero "lejos de constituir una formación moderna, artificial, el vínculo de la tecnología con el conocimiento es muy antiguo”, corrige Layton (1974, p. 31).

Es que, ya al reparar en la etimología, notamos que la obvia derivación del término tecnología de la expresión griega tekhne indica un fenómeno que pertenece al ámbito del conocimiento. En efecto, la tekhne no era un mero hacer, sino un saber hacer. Mitcham (1994, p. 118) recuerda que en Platón tekhne y episteme estaban estrechamente asociadas y que Aristóteles define la tekhne como un hábito que implica un logos, diferenciándose de la episteme porque versa sobre lo que es mutable. Naturalmente, consideraciones etimológicas difícilmente son probatorias, pero a menudo proporcionan buenas pistas. En todo caso, es ciertamente más seguro recurrir a consideraciones históricas y teóricas para verificar que la tecnología es algo diferente de ciencia aplicada.

Reparemos en que, si así no fuera, deberíamos retirar de su dominio realizaciones complejas de otras épocas, como las pirámides elevadas por diversos pueblos, los acueductos romanos y las catedrales medioevales, construcciones todas que no pudieron utilizar conocimientos científicos en el sentido moderno de las palabras. Deberíamos ignorar también el uso de ciertas drogas (efedrina, cocaína, quinina, curare) en la medicina de diversas culturas, así como muchos procesos industriales precientíficos (la fabricación del queso, la fermentación, las tinturas...) (cf. Feibleman, 1983 [1961], p. 36). Por otra parte, numerosas invenciones no se originaron en la aplicación deliberada de conocimientos científicos ni fueron realizadas por científicos. Uno de los casos más conocidos es la invención de la máquina de vapor, que en vez de ser el resultado de teorías científicas contribuyó a desarrollar la termodinámica.

Tal vez la principal dificultad para reducir la tecnología a pura aplicación de la ciencia resida en la propia índole del conocimiento científico. Peter Kroes (1989, p. 377) llama la atención sobre el hiato (gap) existente entre los resultados de la ciencia básica y los conocimientos necesarios para fines tecnológicos, debido al amplio alcance de las teorías y al uso de idealizaciones, lo que obliga, en todo caso, a adaptar el conocimiento científico para posibilitar su aplicación. A su vez, los tecnólogos desarrollan teorías de aplicación limitada, porque - y aquí notamos otra diferencia - el conoci-

1 Por ejemplo Singer et al. (1954) e Daumas (1962-1968). Layton observa que las obras de historiadores norteamericanos (Lynn White, Derek Price, entre otros) son más sensibles a la peculiaridad de la tecnología, pero no consiguieron evitar que "el énfasis en la técnica tuviera un efecto distorsionador" también en los EUA (Layton, 1974, p. 33). 
miento tecnológico es específico para una determinada tarea, un aspecto enfatizado por Joseph C. Pitt (2001, p. 38). Sin embargo, hablar de "adaptación” del conocimiento científico para fines tecnológicos es insuficiente, pues la tecnología implica siempre invención. Joseph Agassi subraya que la ciencia aplicada es "un ejercicio de deducción" a partir de la ciencia pura, pero que "existe una brecha entre la ciencia aplicada y la implementación de sus conclusiones, una brecha que debe ser salvada por la invención" (1974 [1966], p. 52).

Las precisiones anteriores corresponden a la circunstancia de que la tecnología es una actividad dirigida a la producción de algo nuevo y no al descubrimiento de algo existente. El conocimiento tecnológico configura por eso un área sui generis, la de las "ciencias de lo artificial", explorada por Herbert A. Simon en un libro ya clásico (Simon, 1981 [1969]). Lo artificial constituye un sistema adaptado al ambiente en función de determinado propósito humano, un objeto (artefacto) con propiedades deseadas, ideado y fabricado conforme un diseño o proyecto (design). ${ }^{2}$ Por eso, puede ser llamado "conocimiento prescriptivo" (cf. Vincenti, 1990) en contraste con el conocimiento descriptivo procurado por la ciencia. Además, al ser una actividad productiva, la tecnología enfrenta problemas que no afectan al científico básico, como los relativos a la factibilidad, la confiabilidad y la eficiencia de los inventos, a la relación costo-beneficio etc., para los que la ciencia no ofrece soluciones listas (cf. Kroes, 1989, p. 377).

Pero existen también nociones distintivamente tecnológicas, comenzando por la idea de la máquina, desde los instrumentos primitivos hasta los aparatos automáticos, conceptos como los de "switch" y "optimización”, y teorías enteras como las de la cibernética, la hidrodinámica y la teoría de las redes (cf. Mitcham 1994, p. 95). A propósito de las teorías tecnológicas, Bunge señala la existencia de dos tipos: substantivas y operativas. Las primeras proporcionan conocimiento sobre la acción tecnológica (por ejemplo, una teoría sobre el vuelo); las operativas tratan de las acciones de que depende el funcionamiento de los artefactos, como, por ejemplo, una teoría de las decisiones óptimas sobre la a distribución del tráfico aéreo en una región dada (Bunge, 1969, p. 684). Joseph Pitt (cf. 2000, p. 33 ss.) marca otra diferencia al afirmar que el conocimiento científico está "limitado por la teoría" (theory bounded, una expresión acuñada por W. Vincenti, 1990), mientras que el conocimiento tecnológico está "especificado por la tarea" (task specific).

2 Cabe aclarar que, aunque la mayoría de los estudiosos de la tecnología se refieren a lo artificial como si fuese un campo de objetos, de hecho existen también materiales artificiales (por ejemplo, el plástico) y procesos artificiales (como la contención del caudal de un río por una represa). Bunge trata adecuadamente de esa variedad y define lo artificial como "toda cosa, estado o proceso controlado o hecho deliberadamente con ayuda de algún conocimiento aprendido, y utilizable por otros" (Bunge, 1985b, p. 33-4). 
Si las teorías tecnológicas son diferentes de las científicas, otro tanto puede decirse de los datos con que trabaja el tecnólogo, pues estos últimos provienen en su mayoría de la experiencia no científica (siendo particularmente importantes para adaptar el artefacto a sus circunstancias efectivas de funcionamiento). Sin embargo, la singularidad del conocimiento tecnológico se aprecia más aún al reparar que exige datos relativos a exigencias (técnicas, económicas, culturales) que el artefacto debe satisfacer (cf. Vincenti, 1990, p. 216-7). A las diferencias hay que agregar que la ciencia busca establecer leyes que "gobiernan" los fenómenos naturales, mientras la tecnología formula reglas de acción para dar origen a los fenómenos artificiales. Bunge (1969, p. 694 ss.) defiende que las reglas derivan de las leyes ("enunciados nomológicos"), o se apoyan en ellas, mediante "enunciados nomo-pragmáticos". 3

Otro aspecto en que difieren la ciencia y la tecnología consiste en que en esta última se apela mucho más al pensamiento analógico y visual que en aquella, sin excluir, es claro, el pensamiento abstracto y verbal (cf. Vincenti, 1990, p. 221; Baird, 2004, p. 62). El pensamiento analógico es particularmente útil al adaptar recursos de un artefacto ya existente en la producción de otro nuevo, y "proyectistas [designers] destacados son invariablemente destacados pensadores visuales" (Vincenti, 1990, p. 221). Simon (1981 [1969], p. 18-21) describe la producción tecnológica como "conocimiento por simulación" (hoy casi trivializado por el uso de la computadora). Naturalmente, la simulación no es ajena al proceder de la ciencia, pero los modelos tecnológicos se diferencian porque las variables a ser consideradas e incorporadas al modelo vienen dictadas por la meta a alcanzar, mientras que en la ciencia el criterio de selección no es tan específico. ${ }^{4}$ También los experimentos tecnológicos son diferentes de los científicos, pero no porque en aquéllos no se busque conocimiento, sino porque se busca un

3 Por ejemplo, la ley científica según la cual "El magnetismo desaparece por encima de la temperatura de Curie" (en forma condicional: "si la temperatura de un cuerpo imantado rebasa su punto de Curie, entonces el cuerpo pierde su imantación") fundamenta el enunciado nomo-pragmático: "si se calienta un cuerpo imantado por encima de su punto de Curie, entonces pierde su imantación”, que a su vez fundamenta dos reglas tecnológicas: "para desimantar un cuerpo, caliénteselo por encima de su punto de Curie", y "Para evitar la desimantación de un cuerpo, no se lo mantenga por encima de su punto de Curie" (cf. Bunge, 1969, p. 696). Pero no hay que creer que la derivación de reglas a partir de leyes es puntual ni mecánica. Eso significaría ignorar el aspecto creativo de la tecnología (cf. Kroes, 1998, p. 8). En todo caso, la tesis de Bunge puede entenderse en el sentido de que no hay regla eficaz que no tenga respaldo en leyes.

4 Las variables a ser consideradas en el fenómeno dependen de (y caracterizan), según los casos, la disciplina (física, química etc.), la teoría (mecánica clásica o cuántica), o el enfoque (estructuralista, marxista etc.). En lo que respecta al condicionamiento general de la producción de artefactos (de que se derivan las variables interesantes), Bunge (1985a, p. 225) cita: "no violar las leyes naturales, ser factible, operar de manera eficaz y confiable, tener un costo que no exceda determinados valores y producir beneficios que (idealmente, al menos) superen los efectos laterales indeseables". 
conocimiento diferente. El artefacto, ¿funcionará? ¿Habrá acaso factores no previstos teóricamente que serán detectados experimentalmente? Etc.

Igualmente diferentes son las explicaciones tecnológicas. Kroes (1998, p. 3) hace notar que el objeto tecnológico posee, en cuanto objeto físico, una estructura, pero que su carácter de artefacto le viene de la función que le es atribuida. Por esa (simple) razón, la explicación de un objeto tecnológico no puede equivaler a su explicación causal. La descripción física de la estructura del objeto da razón de por qué opera de ese modo, pero no de que su función sea ésta o aquélla. Es el diseño (design) o proyecto el que encierra la explicación tecnológica: muestra de qué modo, en términos de su estructura física, el artefacto desempeña determinada función. Esa diferencia puede ser destacada también diciendo que la tecnología implica descripciones funcionales que no se deducen de descripciones estructurales (Kroes, 2001, p. 3-4). Tratase de una diferencia que se origina en el propósito de la tecnología: mientras la ciencia aspira a entender la realidad, la tecnología se propone controlarla.

La propia noción de conocimiento parece alterarse en el campo de la tecnología. Skolimowski (1983 [1966], p.44) lo caracteriza como conocimiento "de lo que está por ser" (what is to be), en sintonía con H. A. Simon (1981, Cap. 1), que lo describe como conocimiento de lo posible. Kroes (2001, p. 2-3) lo denomina "conocimiento de naturaleza funcional", incluyendo conocimiento de la naturaleza física, de relaciones medios-fines y de la acción conveniente. 5 Este mismo autor observa que los enunciados tecnológicos admiten por eso una apreciación de su verdad o falsedad diferente de la de los enunciados científicos. Se puede afirmar que un artefacto desempeña bien o mal su función (siendo esta afirmación verdadera o falsa, según el caso), mientras que no cabe hacer la misma afirmación a propósito de una estructura física. Vale decir que el conocimiento tecnológico incluye, además de enunciados descriptivos, expresiones normativas (cf. Simon, 1981 [1969], p. 8; Vries, 2003).

Debido a la especificidad del conocimiento tecnológico, algunos autores se inclinan a abandonar la tradicional definición del conocimiento como "creencia verdadera justificada". Joseph C. Pitt (2000, Cap. 1) adopta una perspectiva pragmatista, defendiendo que las reivindicaciones individuales de conocimiento deben ser refrendadas comunitariamente, teniendo como criterio el éxito de la acción. Podríamos decir que el conocimiento consiste en la información colectivamente aceptada y eficaz. Para Pitt, el conocimiento tecnológico resulta más confiable que el científico (cf. Pitt, 2001, p. 6). Este último, dependiendo de teorías, cambia con el cambio de ellas. Por otra parte, la teoría limitativa de su validez no indica lo que debe ser hecho a partir de 
ella. El conocimiento tecnológico, dirigido a una tarea específica, tiene como resultado tipos de soluciones que son registradas en obras de referencia por los ingenieros. Aunque esta especie de conocimiento sea a veces considerado desdeñosamente como "ingeniería de libro de cocina", señala Pitt, es en cierto modo superior al científico en certeza y eficacia. ${ }^{6}$

Este enfoque pragmatista culmina en el análisis de Davis Baird (2002; 2004), que propone una "epistemología material", sosteniendo que los objetos (principalmente, los instrumentos) que producimos encarnan nuestro conocimiento del mundo de manera análoga a como lo hacen las palabras. Para este autor, los artefactos (un reloj, un motor etc.) son portadores de conocimiento tanto como las teorías. ${ }^{7}$ La verdad "material" de este "conocimiento-cosa" (thing knowledge) está dada por el desempeño satisfactorio de la función atribuida al objeto. Baird está convencido, no sólo de la realidad y peculiaridad del saber tecnológico, sino de que el mismo constituye una vía de acceso a nuestras capacidades de comprender la realidad diferente de la representada por el lenguaje (cf. 2004, p.4,0). Y aunque ve su epistemología material como emparentada con la teoría popperiana del "conocimiento objetivo" (el reino de las teorías y problemas, que existiría en cierta autonomía con relación al mundo material y a las mentes humanas), cree más correcto afirmar que el "conocimiento-cosa" y el "conocimiento objetivo" son instancias que interactúan, ambas, con los mundos material y psíquico (p. 144). Baird entiende que comprender el conocimiento objetivo ayuda a comprender el conocimiento subjetivo (esto es, las creencias de los sujetos) y viceversa.

No obstante, otros autores conservan la noción de creencia verdadera justificada para el conocimiento tecnológico, y discriminan diversas formas o modalidades del mismo. Sintetizando contribuciones de diversos autores, Mitcham (cf. 1994, p. 193-4) distingue, dentro del saber tecnológico, las habilidades sensorio-motrices, las máximas teóricas, las leyes descriptivas (o reglas tecnológicas) y las teorías tecnológicas. Las habilidades pertenecen, obviamente, al ámbito del "saber hacer" (know how) y del conocimiento tácito (en el sentido de Polanyi), y no del "saber que". Son adquiridas por entrenamiento, imitación, ensayo y error. Las reglas tecnológicas tie-

6 En cambio, desde una perspectiva realista, Bunge considera las teorías tecnológicas más ricas desde el punto de vista práctico pero más pobres desde el punto de vista teórico, precisamente porque el tecnólogo está menos interesado en la verdad que en la eficiencia (Bunge, 1969, p. 686).

7 Baird distingue tres tipos de instrumentos: los que representan el objeto conocido, como los modelos materiales (desde los "planetarios" del siclo xix al modelo helicoidal del ADN); los que encierran "conocimiento que funciona" (working knowledge), como la bomba de aire o el ciclotrón; y los que constituyen "conocimiento encapsulado", que son los instrumentos de medida, desde una regla hasta un espectrómetro (Baird, 2004, caps. 2, 3 y 4). 
nen su fundamento en conocimientos científicos. Las teorías tecnológicas son las instancias tecnológicas más próximas de la noción tradicional de conocimiento, aunque más nítidamente en el caso de las "teorías substantivas” (Bunge, 1969, p. 684). En todo caso, Mitchan comenta que la proximidad de las modalidades del saber tecnológico a la noción clásica ("creencia verdadera justificada") se puede entender en el sentido de que "las creencias relativas a la fabricación y el uso de artefactos pueden ser justificadas recurriendo a habilidades, leyes, reglas o teorías" (Mitchan, 1994, p. 194). ${ }^{8}$ Ya sea entendido en sentido pragmático o en sentido teórico, el conocimiento tecnológico parece tener una índole claramente sintética o integradora, a diferencia del carácter más analítico de la ciencia básica (cf. Hummon 1984, p. 70; Constant, 1984, p. 34).

Por otra parte, la producción tecnológica está, obviamente, lejos de ser una actividad casual. Bunge (1985a, p. 236) asimila el método tecnológico al método científico general. Como es notorio, Bunge es un defensor' 9 de la existencia de una metodología básica válida para toda investigación científica (lo que él llama su "estrategia”), que en el caso de la tecnología consiste en el ciclo: problema práctico-proyecto-prototipoprueba-eventual corrección del proyecto o reformulación del problema). ${ }^{10}$ El proyecto (design) es en cierto modo el corazón del procedimiento tecnológico y Bunge aclara que no defiende la existencia de un método para proyectar: la idea de que tal método exista le parece "tan absurda como la idea de que todo lo que se debe hacer para ser un científico es dominar el método científico" (Bunge, 1985a, p. 228). Lo que Bunge sostiene es que hay una secuencia lógica mínima en toda actividad productora de tecnología (así como en la investigación científica). Además, Bunge subraya el ingrediente científico del proyecto tecnológico, definiéndolo como "la representación anticipada de un artefacto con el auxilio de algún conocimiento científico" (Bunge, 1985a, p. 225). Los desafíos de esa actividad fueron analizados por Walter Vincenti (1984), en un libro ya clásico, que he mencionado varias veces. Como ingeniero, Vincenti llama la atención sobre los aspectos empíricos, prácticos, imprevisibles de la producción tecnológica, y sobre las diversas formas y circunstancias en que el conocimiento científico, sin que se pueda prescindir de él, es insuficiente para resolver problemas tecnológicos, debe ser adaptado a las dificultades prácticas o tiene una relevancia diferente para

8 Existen, agrega oportunamente Mitcham, diferentes interpretaciones de esa justificación: realista, instrumentalista, pragmatista y los tecnólogos con facilidad adhieren a la realista (Mitchan, 1994).

9 Defensa rara en una época en que se está volviendo un lugar común rechazar esa pretensión y hasta considerarla absurda.

10 Naturalmente, quedan fuera de esta comparación las etapas posteriores, no cognitivas, de la producción tecnológica (fabricación en serie, monitoración etc.). 
el técnico y para el científico. ${ }^{\mathbf{1 1}}$ De particular interés son las conclusiones de Vincenti sobre categorías de conocimiento tecnológico y actividades generadoras de conocimiento tecnológico. Las categorías mencionadas son: conceptos fundamentales del design (como el "principio operacional" - una noción acuñada por Polanyi - que define un dispositivo tecnológico o la "configuración normal" de un aparato), ${ }^{\mathbf{1 2}}$ criterios y especificaciones (las metas generales y cualitativas a ser alcanzadas deben ser transformadas en metas específicas y cuantitativas, técnicamente definidas), herramientas teóricas (desde teorías y métodos matemáticos a conceptos puramente técnicos, como feedback), datos cuantitativos (que a diferencia de la ciencia no son solamente descriptivos, sino también prescriptivos, o sea establecen requisitos a satisfacer), consideraciones prácticas (know how de diverso tipo, proveniente de la experiencia profesional), e "instrumentos para proyectar" (procedimientos como la optimización, modos de pensar que se mostraron eficaces y, sobre todo, habilidad para juzgar) (Vincenti, 1990, p. 208 ss.). Las actividades generadoras de conocimientos son, siempre según Vincenti, las siguientes: la transferencia de conocimientos científicos (siempre, de algún modo adaptados); la invención de nociones (en lo que esa actividad tiene de creativo, no programable); la investigación teórica (semejante a la científica, salvo por el direccionamiento al proyecto); la investigación experimental (también semejante a la científica, pero con mayor peculiaridad en el uso de métodos y recursos); ${ }^{\mathbf{1 3}}$ la práctica de proyectar, que revela problemas y necesidades que estimulan la búsqueda de conocimiento; la producción del artefacto, durante la cual pueden hacerse observaciones (por ejemplo, en forma de fallas) que suscitan nuevo saber, y finalmente la prueba directa (direct trial) del producto tecnológico por los constructores y usuarios, diferente de los testes iniciales, pues evidencia problemas o aspectos a mejorar que sólo la experiencia continuada revela.

Un proyecto tecnológico, simple o de grandes proporciones, comienza por la identificación de un problema. "Resolver problemas constituye la mayor actividad cognoscitiva del profesional de la tecnología”, escribe Rachel Laudan (1984,b, p. 84), quien ofrece una interesante taxonomía de los problemas tecnológicos. Un primer tipo con-

11 Los casos analizados por Vincenti corresponden a su área de experiencia (ingeniería aeronáutica) y al diseño "normal", pero el autor argumenta de manera convincente sobre la plausibilidad de ver en sus conclusiones aspectos del design aplicables a otros campos tecnológicos.

12 Vincenti apunta aquí una diferencia "importante" entre ciencia y tecnología, pues aunque el conocimiento científico puede ayudar a comprender un principio operacional (por ejemplo, el principio de vuelo por elevación producido por un rotor en el helicóptero) el conocimiento científico no implica, meramente, el principio. Ciertamente, rebítese aquí la observación de Kroes de que lo característico del dispositivo tecnológico es su función.

13 A veces, recurriendo a experiencias vulgares que no tendrían lugar en la ciencia avanzada, como romper cosas para observar determinados problemas de peso (cf. Vincenti, 1990, p. 232). 
siste en los problemas suscitados directamente por el ambiente y aún no resueltos por ninguna tecnología. Detectar un problema de esos es lo contrario a percibir el curso de los acontecimientos como inevitable. Contrariamente a la suposición vulgar, afirma Laudan, "la percepción inmediata de dificultades raramente provoca una respuesta tecnológica a menos que haya una tecnología disponible que se puede aplicar directamente a la situación, o que puede ser convenientemente modificada" (1984b, p. 85). Eso ocurre porque hace mucho que vivimos en un medio ya tecnológicamente modificado. En consecuencia, "encontramos el locus principal del cambio tecnológico en la modificación de la tecnología existente" (Laudan, 1984b, p. 85). Otro tipo de problemas surge en las fallas funcionales de las tecnologías actuales. Típicamente, cuando un artefacto o sistema es sometido a exigencias mayores de las proyectadas, o cuando es aplicado a nuevas situaciones. Cabe agregar que la existencia de una tecnología no es prueba de que su funcionamiento sea perfecto. Las tecnologías, afirma Laudan, son implementadas tanto por su necesidad como por su desempeño, y menciona las tecnologías médicas anteriores al siglo xx como ejemplos (1984b, p. 86). Un tercer tipo de problemas nace de la extrapolación a partir de características de la tecnología corriente. "El mecánico que construyó con éxito una máquina de hilar de 50 carreteles tiende a ver la producción de una máquina similar con 100 carreteles como su próximo problema, aunque la máquina que construyó no tenga fallas" (1984b, p. 86). Un cuarto tipo de problemas, "y muy importante", responde a la percepción de un desequilibrio entre tecnologías relacionadas. Un ejemplo sería el problema de la estabilidad de los navíos al cambiar de naves de madera movidas por velas a naves de hierro movidas a vapor. Por último, tenemos los problemas tecnológicos adelantados por los conocimientos disponibles, como cuando la teoría aerodinámica previó que aviones impulsados por motores a pistón fallarían a velocidades y alturas superiores a las practicadas hasta entonces (1984b, p. 84). En todo caso, sea cual fuera el origen de los problemas (natural, social, tecnológico), la cuestión de su solubilidad opera siempre como un filtro. Individuos y comunidades tienden a ignorar problemas que parecen insolubles. ${ }^{\mathbf{1 4}}$ La solubilidad es, casi obviamente, función de la experiencia pasada, y en el caso de las comunidades tecnológicas, de lo que puede llamarse su "matriz" (a semejanza de la "matriz disciplinar" kuhniana).

Es que otro aspecto interesante al apreciar la tecnología en su dimensión cognoscitiva, es la constatación de la existencia de paradigmas tecnológicos, a semejanza de los paradigmas científicos. Edward Constant (1984, p. 29) destaca que las comunida-

14. A nivel vulgar, la aparente insolubilidad de un problema lleva a ver los acontecimientos como una fatalidad y a apelar a soluciones sociales. Por ejemplo, una comunidad que vio su aldea arrasada por una inundación y no concibe medios para evitarlo, optará por mudarse de local (Laudan, 1984,b, p. 84). 
des tecnológicas y sus tradiciones son "el locus primario" de tecnología y su progreso. Una tradición depende de una comunidad de practicantes que se reconocen entre sí, y la concepción orientadora que la anima define el significado de los dispositivos y técnicas, establece los cánones que definen la forma de pertenecer a la comunidad y estimula la creatividad del inventor. Las comunidades tecnológicas y sus paradigmas y tradiciones tienen, sin embargo, sus peculiaridades. Las anomalías, en particular, pueden estar representadas, no sólo por fallas funcionales (cuando el sistema producido no funciona en condiciones nuevas o más exigentes), sino también por "anomalías presumibles", que ocurren cuando la ciencia prevé que la tecnología corriente no funcionará en ciertas circunstancias, o que una nueva tecnología lo hará mejor (cf. Constant, 1984, p. 31). ${ }^{\mathbf{1 5}}$ El reverso de esa diferencia está en otra localizada en la práctica “normal": lo que se entiende por alcanzar resultados "satisfactorios" (en términos kuhnianos, resolver bien un "rompecabezas" del área) es más directo en la tecnología, pues el producto debe funcionar tal como fue proyectado. En la ciencia, lo que se entienda por una teoría, una explicación o un testeo satisfactorios puede implicar más examen y discusión (cf. Constant, 1984, p. 37). A pesar de apreciar el enfoque de Constant, Imre Hronzky (1998, p. 1-2) cree que este último no advirtió el papel de los “ejemplares" en Kuhn, por lo que no llegó a destacar la importancia, en los paradigmas tecnológicos, del saber práctico relativo al oficio, una crítica que fue adelantada por G. Gutting (cf. 1984, p. 48 ss.). Hronzky señala más diferencias entre los paradigmas tecnológicos y los científicos. En los primeros, otro tipo de anomalía puede surgir por presiones de tiempo o falta de herramientas, debido a que los tecnólogos, una vez más a diferencia de los científicos, deben atenerse a la tarea que les fue propuesta. Ciencia y tecnología se diferencian también debido a la presencia, dentro de las comunidades tecnológicas, de actores que no tienen paralelo en las comunidades científicas (como los productores y los usuarios de los artefactos). Por eso, la contribución a la producción de nuevo conocimiento (y a un eventual cambio de paradigma) puede originarse en la intervención de legos, no necesariamente de técnicos. Otra diferencia consistiría en que los tecnólogos suelen recurrir a todo tipo de conocimiento disponible (un aspecto mencionado ya por Vincenti): el tecnólogo es una suerte de "bricoleur". Hronzky añade que la comunidad tecnológica es más vaga (fuzzy) que la científica, lo que hace que una anomalía pueda ser percibida más directamente (por ejemplo, en el fracaso del artefacto en manos de los usuarios), pero al mismo tiempo que no sea tan evidente la exigencia de un cambio de paradigma. Hronzky (1998, p.2) observa tam-

15 Como cuando se previeron las limitaciones de los aviones a pistón, como ya fue mencionado. Constant trató del tema en su libro más importante y citado, (1980). 
bién que, aunque tanto las comunidades científicas como las tecnológicas estén compuestas por individuos y por actores grupales (como firmas y laboratorios), estos últimos, como "vehículos y propietarios de saber especializado y tácito", tienen una gravitación en la tecnología más pronunciada que en la ciencia.

Las revoluciones tecnológicas tampoco parecen ser iguales a las científicas. Constant (cf. Gutting, 1984, p. 53) las caracteriza como la adhesión a una nueva tradición tecnológica por una comunidad nueva o redefinida. A diferencia de las revoluciones científicas en el modelo de Kuhn, que son a la vez innovadoras y eliminatorias, las revoluciones tecnológicas no necesitan implicar una elección radical, no suponen forzosamente una nueva comunidad y son compatibles con la continuidad de tecnología "normal". El ejemplo principal de Constant es que la revolución del avión de propulsión a chorro no eliminó repentinamente la fabricación de aviones de motor a pistón. Por su parte, Rachel Laudan argumenta que una revolución tecnológica no siempre responde al estancamiento de una tecnología tradicional (como lo demostraría la transformación - revolucionaria - de la máquina de vapor alternada en rotativa en el siclo XVIII), y que cambios en el ambiente pueden desencadenar revoluciones, como cuando la deforestación inglesa provocó el desarrollo de la tecnología de extracción del carbón mineral (cf. Laudan, 1984b, p. 99). Por otra parte, innovaciones tecnológicas pueden ocasionar revoluciones científicas. Baird (2004, Cap. 5) menciona el caso de la transformación de la química analítica durante el siglo $\mathrm{xx}$, pasando del análisis cualitativo que identifica las substancias por la separación de sus componentes, para el análisis cuantitativo, que las identifica por sus propiedades físicas mediante diversos instrumentos y procedimientos (espectrofotometría, difracción de rayos x, polarografía etc.). El desarrollo de los mismos (y no un cambio de teoría) fue el responsable de una revolución en la química analítica, pues la índole misma de lo que significa esa actividad fue transformada. ${ }^{\mathbf{1 6}}$ No puede dejar de mencionarse que el papel de la tecnología en las revoluciones científicas fue sostenido antes por Derek de S. Price (1984). Para él, la producción y la innovación de instrumentos tuvieron un papel más importante en el origen de revoluciones científicas que el de las ideas. La "revelación artificial" producida por los instrumentos (ver su brillante análisis del telescopio) es, en su interpretación, un factor clave para entender cambios científicos y tecnológicos (Price, 1984, p. 112).

16 Una revolución, aclara Baird, no en el sentido de T. Kuhn (no hubo anomalías ni "inconmensurabilidad"), sino en el de autores como Cohen y Hacking, pues fue algo visto como tal por los propios científicos, tuvo impacto en los tratados y libros de texto, fue juzgada como una revolución por los historiadores, implicó cambios conceptuales, originó nuevas instituciones y hasta produjo cambios sociales. 
El reconocimiento de la peculiaridad del conocimiento tecnológico no significa, es claro, que no tenga semejanzas con el conocimiento científico, además de las mencionadas anteriormente para señalar diferencias. El diseño tecnológico (más específicamente, la actividad de simular) se parece a la actividad de modelar (idear modelos referentes a los mecanismos de producción de los fenómenos), propia de la ciencia. En ambos casos, se trata de ver si el modelo funciona (Cf. Simon, 1981, p. 17 ss.). Hans Jonas (1983 [1966], p. 336) propuso interpretar la ciencia moderna como dirigida a comprender "la naturaleza al trabajo" (Nature at work) viéndola así como inherentemente tecnológica, una concepción defendida en detalles por Joseph Rouse (1994 [1987]) al sostener la continuidad entre el experimento científico y las aplicaciones tecnológicas. En el mismo sentido, Hugh Lacey (1998) ve la ciencia moderna como basada en "estrategias materialistas" que enfocan los fenómenos tan sólo en términos de las propiedades que los vuelven susceptibles de ser controlados. Esta tendencia encuentra su extremo en autores que ven la ciencia como una forma de tecnología. Tal es el caso de James K. Feibleman (1982), que entiende la ciencia como "la clase de tecnología que se dirige, no a problemas prácticos, sino a los teóricos". ${ }^{17}$ Menos radical, Kroes (1989, p.379), después de haber apuntado las diferencias, manifiesta su opinión de que hay una transición gradual del conocimiento científico al tecnológico. Ferré (1995 [1988], Cap. 4) ve en la ciencia y la tecnología “dos mellizas no idénticas", pues ambas responderían a la capacidad humana de pensar (inteligencia), en la medida en que ésta última se diferencia en inteligencia práctica (capacidad de sobrevivir resolviendo problemas) e inteligencia teórica o razón (capacidad de comprender el mundo). Desde la Modernidad, argumenta Ferré, la razón se puso al servicio de la capacidad de sobrevivir, surgiendo la "inteligencia práctica teoréticamente fundamentada".

En verdad, junto con la reivindicación de la peculiaridad del conocimiento tecnológico, se da actualmente atención al parecido de la ciencia con la tecnología (tal como se aprecia en algunas de las referencias anteriores), debido a la creciente intervinculación de ambas actividades y su aparente fusión en la llamada "tecnociencia". Ramón Queraltó (2001) sostiene que la tecnología se ha convertido en una mediación entre la ciencia y la realidad, dejando de ser mero instrumento. Ese carácter se percibiría en el creciente predominio de la ciencia aplicada sobre la pura y de la "verdad

17 "Comenzando con herramientas e instrumentos construidos por su utilidad en tareas prácticas, hubo sólo una breve distancia lógica hasta los instrumentos inventados por lo que ellos podían descubrir acerca de la naturaleza de las cosas" (Feibleman, 1982, p. 8). 
pragmática” (¿para qué sirve un objeto?) sobre la "verdad teórica” (¿qué es ese objeto?). Queraltó añade que se da un condicionamiento recíproco entre la teoría y los testeos. Antes, el testeo estaba completamente subordinado a las ideas a ser comprobadas. Ahora, los medios tecnológicos condicionan lo que será testeado. El autor ejemplifica con el caso del ciclotrón: las partículas son destruidas para estudiarlas, lo que genera nuevos “objetos”. Por fin, las manipulaciones tecnológicas entran hoy decisivamente en la elaboración del "objeto de conocimiento", principalmente porque las propiedades a ser conocidas se reducen a las vinculadas a aquellas manipulaciones. (Nótese la semejanza con la posición de Lacey.)

El parecido de la ciencia moderna con la tecnología se revela también en la ambigüedad de ciertas disciplinas. Alberto Cordero (2001, p. 130) cita casos como el de la "medicina darwinista", la ciencia de los materiales o la nanotecnología para mostrar que actualmente es difícil clasificar ciertas disciplinas "de punta". ${ }^{8}$ Admitiendo la dificultad de diferenciar ciencia y tecnología en función de objetivos, métodos y contextos (en muchas investigaciones actuales, es difícil justificar la diferencia), el autor rechaza también la "tentación pantecnológica" de explicar completamente la ciencia por la tecnología. ${ }^{19}$ Cordero observa que ciencia y tecnología comparten conjuntos semejantes de metas y criterios de selección, pero que "los valores o pesos vinculados a aquellos elementos comunes se diferencian manifiestamente en las dos formas de actividad". Eso significa, por ejemplo, que la búsqueda de conocimiento no es exclusiva de la ciencia, y que la búsqueda del éxito no es privativa de la tecnología. Pero en la tecnología, el criterio de éxito generalmente subraya la satisfacción de deseos o necesidades no epistémicos. En la ciencia, se busca satisfacer requisitos epistémicos (como verdad y justificación). Eso porque un objetivo primario de la ciencia es la adquisición de conocimiento, lo que en la tecnología es raramente (si lo es alguna vez) un objetivo primario. Además, la ciencia es “epistémicamente más ambiciosa”, considerándose exitosa si alcanza algo que merezca ser considerado conocimiento verdadero o aproximadamente verdadero. "Las disciplinas tecnológicas pueden ser satisfechas (y habitualmente lo son) con mucho menos" (Cordero, 2001, p. 136). Por fin, también en el plano ético hay diferencias de énfasis: mientras la honestidad en los informes es muy

18 Schummer (2001) muestra que la química preparativa, que ocupa la inmensa mayoría de las investigaciones (más del 90\%), debería ser considerada una tecnología, pues el 95\% de las substancias hoy conocidas son artefactos. Además, perfeccionar la capacidad de producir nuevas substancias se volvió en la química un fin en sí mismo. 19 Una tendencia estimulada por la epistemología de J. Dewey, apunta Cordero (2001, p. 133). Sin embargo, no todo en esa epistemología es criticable, añade, y él mismo se sirve de ella al proponer su propia interpretación de la relación entre ciencia y tecnología. 
importante en la ciencia, en la tecnología hay más motivos aceptables para mentir. Los ejemplos de Cordero (como mentir piadosamente a un paciente en la práctica de la medicina) no parecen relacionados con el aspecto cognitivo de la tecnología. Con todo, podemos imaginar razones socialmente aceptables para mentir (ocultar, disimular etc.) a propósito de proyectos tecnológicos, como la de proteger intereses económicos o evitar consecuencias sociales derivadas de un conocimiento inoportuno de un proyecto. El secreto industrial, el patentamiento de inventos etc. son asuntos relacionados con esta diferencia de ethos entre ciencia y tecnología. Todo eso, para Cordero, "es cuestión tan sólo de moderado grado o énfasis”. En resumen, para él las disciplinas científicas y las tecnológicas tienden a agruparse en torno de diferentes "núcleos de evaluación", por lo que el perfil del entendimiento (understanding) del mundo varía en las dos actividades. Además, el autor aclara que no concibe esa diferencia como algo a priori o fijo, sino histórico. La ciencia y la tecnología "fueron llevadas a un estado de profunda complementariedad, siendo ahora más interdependientes que nunca”, pero eso no significa que siempre hayan sido así, o que deban continuar siéndolo (Cordero, 2001, p. 137).

\section{IV}

Aunque no es mi propósito abordar en este artículo una posible solución a los problemas de filosofía de la ciencia mencionados al comienzo, creo útil consignar algunas conclusiones sobre la dimensión cognoscitiva da la tecnología que puedan ayudar a abordarlos. Estas conclusiones surgen de las lecturas de que extraje la visión de conjunto del conocimiento tecnológico que acabo de ofrecer.

En primer lugar, parece estar fuera de dudas de que la tecnología es un modo específico de conocimiento y, previamente, un modo específico de resolver determinados problemas de conocimiento. Como dice Gary Gutting, ella no es sinónimo de ciencia aplicada, pero tampoco se reduce a técnicas (por más sofisticadas que fueran) sin valor cognitivo, sino que constituye "un cuerpo de conocimiento práctico" (Gutting, 1984, p. 64). La tecnología no se propone, en principio, la obtención de conocimientos por el valor que éstos tengan en un contexto puramente teórico. Busca, ciertamente, el saber útil, pero eso no excluye que produzca ocasionalmente un saber no inmediatamente útil (cf. Gutting, 1984, p. 63).

En todo caso, el conocimiento producido por la tecnología es más "amorfo" y menos "auto-contenido" que el de la ciencia (cf. Weingart, 1984, p. 115 ss.). Las comunidades tecnológicas son menos autónomas, desde el punto de vista cognoscitivo, que las comunidades científicas, pues por la índole de su propósito definidor (producción 
de artefactos) están marcadas por "complejos de orientación”20 de diverso tipo: técnico-científicos, económicos, políticos, culturales. Es esa diversidad de factores que le da el carácter "amorfo" (yo diría ambiguo o complejo) a toda pieza de saber tecnológico, permitiéndole responder a diversas exigencias, de las cuales la verdad es tan sólo una. ${ }^{21}$ Otra manera de apuntar para el mismo fenómeno consiste en mostrar que la relación entre valores cognoscitivos y valores sociales (en sentido amplio) es diferente en ciencia y en tecnología.

Aunque lo anterior sea patente, no debe pasar desapercibido que la tecnología tiene su propia dinámica, su propia lógica operacional. Eso significa que, por más importantes que sean las exigencias económicas y políticas (principalmente) a que un producto tecnológico deba responder, existen siempre condiciones y limitaciones técnicas que no pueden ser ignoradas (Vincenti, 1990, p. 204-5). Laudan observa, muy agudamente, que a menos que se comprenda eso, no se puede entender la manera como los tecnólogos reaccionan a las presiones económicas y sociales, ni como su trabajo afecta la sociedad (Laudan 1984a, p. 4). Significa también que los valores específicamente tecnológicos (como la factibilidad o la economía de recursos) pueden adaptarse a las presiones sociales sólo hasta cierto punto.

Tampoco debe reducirse la tecnología al saber-hacer (know how) y a su ejercicio tácito, como si los únicos momentos de conocimiento explícito y descriptivo correspondieran al ingrediente científico. Desde la Revolución Industrial, observa Laudan (1984a), la proporción del conocimiento tecnológico explícito ha crecido mucho (como lo atestigua la creación de la carrera de ingeniero). Como hemos visto, es imposible ignorar la existencia de teorías y explicaciones específicamente tecnológicas. Si ellas no pueden sustituir el conocimiento tácito y práctico es por la misma razón que tampoco la ciencia excluye estas últimas formas de saber.

Si queda claro que la tecnología no constituye una mera prolongación de la ciencia, pura o aplicada, eso es un obstáculo para concebir la ciencia como inherentemente tecnológica. La complejidad de motivaciones e influencias presente en las creaciones tecnológicas dificulta entenderlas como mera manifestación de un conocimiento del mundo producido por la "voluntad de poder". Es verosímil que en la ciencia básica se haya privilegiado modernamente los aspectos de la realidad que la vuelven controlable (cf. Lacey, 1998), de tal modo que, probablemente, la mayoría del conocimiento de que disponemos hoy en día, si no representa ciencia aplicada, es ciencia aplicable.

20 Esos "complejos" que orientan la investigación incluyen códigos de ética profesional, convicciones disciplinarias, definiciones de áreas de pesquisa y hasta conjuntos de problemas, según Weingart (1984, p. 116).

21 Por otra parte, la utilidad no es el único valor perseguido al identificar un problema tecnológico. Pesan también ocasionalmente motivaciones estéticas e intelectuales, reconoce Laudan (1984a, p. 8-9). 
O que el desarrollo tecnológico (que incluye la manera tecnológica de pensar y de vivir $)^{22}$ fomenta cada vez más el conocimiento científico aplicable. Nada de eso excluye formulación de cuestiones teóricas por mera curiosidad, o como consecuencia del conocimiento ya disponible, ni invalida el hecho de que haya, también, constataciones, teorías y explicaciones que no tienen aplicación o interés práctico. Mejor dicho: conocimientos que un determinado propósito práctico aún no pensó en adoptar y adaptar. Esa intervención circunstancial me parece decisiva para comprender la ciencia y la tecnología sin confundirlas.

Agradecimientos. Este trabajo ha sido financiado por el CNPq, al que agradezco.

\author{
Alberto Cupani \\ Profesor Titular del Departamento de Filosofia \\ de la Universidade Federal de Santa Catarina, Brasil. \\ Investigador del CNPq. \\ cupani@cfh.ufsc.br
}

\begin{abstract}
Philosophy of technology, a rather recent discipline, has been growingly emphasizing the peculiar nature of technological knowledge, mainly the fact that it is different from scientific knowledge. In this paper, I rejoin several characteristics of technological knowledge which are scattered in the literature. I aim thereby at contributing to a better understanding of technological knowledge as a whole and to the solution of some questions regarding the nature of science, in particular its possible intrinsic relation to technology.
\end{abstract}

KEYwords $•$ Technological knowledge. Technology as knowledge. Scientific knowledge. Control of nature.

22 Me refiero a la actitud para la cual todo problema humano puede reducirse a una cuestión de encontrar los medios adecuados para alcanzar determinado fin (cf. Borgmann, 1984). 


\section{REFERENGIAS BIBLIOGRÁFICAS}

Agassi, J. The confusion between science and technology in the standard philosophy of science. In: RAPP, F. (Ed.). Contributions to a philosophy of technology. Dordrecht: D. Reidel, 1974 [1966]. p. 40-59.

BAIRD, D. Thing knowledge: function and truth. Techné: Journal of the Society for Philosophy and Technology, 6, 2, p. 13-27, 2002. Disponible en: <http://scholar.lib.vt.edu/ejournals/SPTP/v6n2/baird.html>.Acceso en: 22 jun. 2005 .

. Thingknowledge. A philosophy of scientific instruments. Berkeley: University of California Press, 2004. Borgmann, A. Technology and contemporary life. Chicago: The University of Chicago Press, 1984.

Bunge, M. La investigación científica. Barcelona: Ariel, 1969.

Epistemologia. Um curso de atualização. São Paulo: T. A. Queiros/EDUSP, 1980.

Treatise on basic philosophy. Part II. Boston: D. Reidel, 1985 a. v. 7 .

Seudo ciencia e ideología. Madrid: Alianza, 1985 b.

Constant, E. W. The origins of the turbojet revolution. Baltimore/London: John Hopkins University Predd, 1980.

. Communities and hierarchies: structure in the practice of science and technology. In: LaUdAN, R.

(Ed.). The nature of technological knowledge. Are models of scientific shange relevant? Dordrecht: D. Reidle, 1984 . p. $27-46$.

Cordero, A. On the growing complementarity of science and technology. In: LenK, H. \& Maring, M. (Ed.). Advances and problems of the philosophy of technology. Münster: LIT Verlag, 2001. p. 129-4,O.

Daumas, M. Histoire general des techniques. Paris: PUF, 1962-1968. $5 \mathrm{v}$.

Feibleman, J. K. Technology and reality. New York: The Hague/Martinus Nijhoff, 1982.

Pure science, applied science, and technology: an attempt at definition. In: Мiтснам, C. \& Mackey,

R. (Ed.). Philosophy and technology. Readings in the philosophical problems of technology. New York: The Free Press, 1983 [1961]. p. 33-41.

Ferré, F. Philosophy of technology. Athens/London: The University of Georgia Press, 1995 [1988].

GaVroglu, K. et al. (Ed.). Imre Lakatos and theories of scientific change. Dordrecht: Kluwer, 1989.

Gutting, G. Paradigms, revolutions, and technology. In: Laudan, R. (Ed). The nature of technological knowledge. Are models of scientific change relevant? Dordrecht: D. Reidel, 1984. p. 47-66.

Нвоnzку, I. Technological 'paradigms': cognitive traditions and communities in technological change. Paideia. Boston: s/n, 1998. (Publicación de los trabajos del XX Congreso Internacional de Filosofía). Disponible en: 〈http://www.bu.edu/wcp/Papers/Scie/ScieHron.htm>. Acceso en: 29 jun. 2005.

Hummon, N. P. Organizational aspects of technological change. In: Laudan, R. (Ed.). The nature of technological knowledge. Are models of scientific change relevant? Dordrecht: D. Reidel, 1984. p. 67-82.

Jarvie, J. Technology and the structure of knowledge. In: Mitcham, C. \& Mackey, R. (Ed.). Philosophy and technology. Readings in the philosophical problems of technology. New York: The Free Press, 1983 [1967]. p. $54-61$.

Jonas, H. The practical uses of theory. In: Mitcham, C. \& Mackey, R. (Ed.). Philosophy and technology. Readings in the philosophical problems of technology. New York: The Free Press, 1983 [1966]. p. 335-46.

Kroes, P. Philosophy of science and the technological dimension of science. In: GAVRogLu, K. et al. (Ed.). Imre Lakatos and theories of scientific change. Dordrecht: Kluwer, 1989. p. 375-81.

. Technological explanations: the relation between structure and function of technological objects. Society for Philosophy and Technology, 3, 3, p. 1-9, 1998. Disponible en: <http://scholar.lib.vt.edu/ ejournals/SPTP/v3n3/kroes.html>. Acceso en: 23 jun. 2005 .

Technical functions as dispositions: a critical assessment. Techné: Journal of the Society for Philosophy and technology, 5, 3, p. 1-16, 2001. Disponible en: <http://scholar.lib.vt.edu/ejournals/SPTP/ט5n3/ kroes.html>. Acceso en: 22 jun. 2005 . 
Lacey, H. Valores e atividade científica. São Paulo: Discurso, 1998.

LaUdan, R. (Ed.). The nature of technological knowledge. Are models of scientific change relevant? Dordrecht: D. Reidel, 1984 .

. Introduction. In: LaUdAn, R. (Ed.). The nature of technological knowledge. Are models of scientific change relevant? Dordrecht: D. Reidel, 1984a. p. 1-26.

. Cognitive change in technology and science. In: LaUdan, R. (Ed.). The nature of technological knowledge. Are models of scientific change relevant? Dordrecht: D. Reidel, 1984, b. p. 83-104.

LaYton, E. W. Technology as knowledge. Technology and Culture 15, 1, p. 31-41, 1974.

Lenk, H. \& Maring, M. (Ed.). Advances and problems of the philosophy of technology. Münster: LIT Verlag, 2001.

Mitcham, C. Thinking through technology. The path between engineering and philosophy. London: The University of Chicago Press, 1994 .

Mitcham, C. \& Mackey, R. (Ed.). Philosophy and technology. Readings in the philosophical problems of technology. New York: The Free Press, 1983.

Pitr, J. C. Thinking about technology. Foundations of the philosophy of technology. New York: Seven Bridges Press, 2000.

. What engineers know. Techne. Journal of the Society for Philosophy and Technology, 5, 3, p. 1-7, 2001. Disponible en: 〈http://scholar.lib.vt.edu/ejournals/SPT/v5n3/pitt.html〉. Acceso en : 23 jun. 2005.

Queraltó, R. Technology as a new condition of the possibility of scientific knowledge. In: Lenk, H. \& MARING, M. (Ed.). Advances and problems of the philosophy of technology. Münster: LIT Verlag, 2001. p. 205-14.

RAPp, F. (Ed.). Contributions to a philosophy of technology. Dordrecht: D. Reidel, 1974 .

Rouse, J. Knowledge and power. Toward a political philosophy of science. Ithaca/London: Cornell University Press, 1994, [1987].

Schummer, J. Challenging standard distinctions between science and technology. In: Lenk, H. \& Maring, M. (Ed.). Advances and problems of the philosophy of technology. Münster: LIT Verlag, 2001. p. 215-30.

Simon, H. A. The sciences of the artificial. Cambridge/Massachussets: The MIT Press, 1981 [1969].

Singer, C. et al. A history of technology. Oxford: Clarendon Press, 1954. 8 v.

Sкоцiмowski, H. The structure of thinking in technology. In: Mitcham, C. \& Mackey, R. (Ed.). Philosophy and technology. Readings in the philosophical problems of technology. New York: The Free Press, 1983 [1966]. p. 42-9.

Vincentr, W. G. What engineers know and how theyknow it. Baltimore/London: The John Hopkins University Press, 1990.

VRIEs, M. J. de. The nature of technological knowledge: extending empirically informed studies into what engineers know. Techné, 6, 3, p. 1-21, 2003.

WEIngart, P. The structure of technological change: reflections on a sociological analysis of technology. In: LAUDAN, R. (Ed.). The nature of technological knowledge. Are models of scientific change relevant? Dordrecht: D. Reidel, 1984. p. 115-42. 